\title{
Assessment of Undiscovered Petroleum Resources of Northern Afghanistan, 2006
}

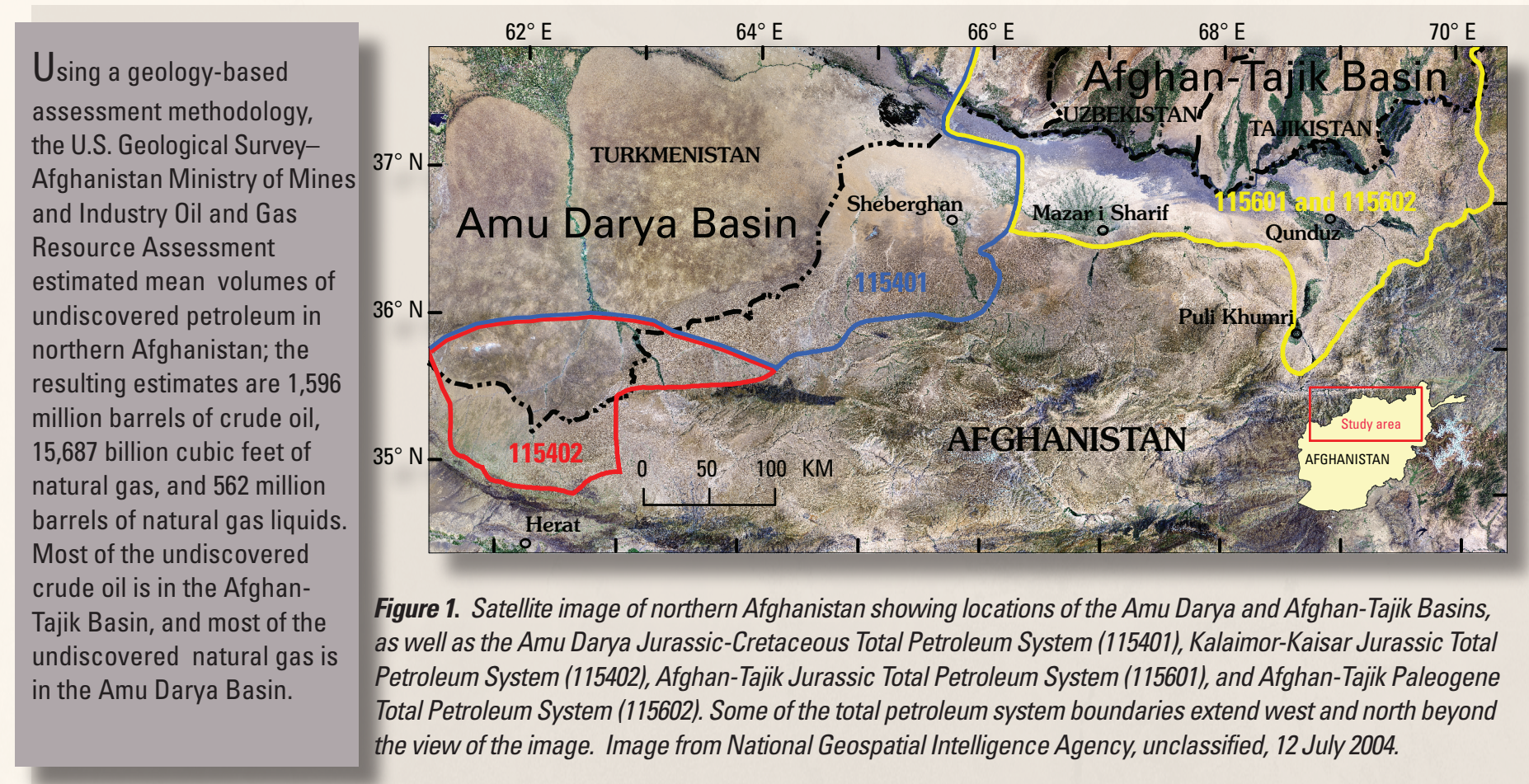

\section{Introduction}

The U.S. Geological Survey (USGS) cooperated with the Afghanistan Ministry of Mines and Industry to assess the undiscovered petroleum resources of Afghanistan. Funding for this effort was provided by the U.S. Trade and Development Agency.

The assessment is based on the geologic elements of a total petroleum system, which include (1) source-rock presence, maturation, petroleum generation, and migration; (2) distribution and quality of reservoir rocks; and (3) character of traps and time of formation with respect to petroleum migration. Data from detailed studies of geochemistry, petroleum geology, geophysics, and tectonics combined with historical exploration and production analyses were used to aid in the estimation of the number and sizes of undiscovered petroleum accumulations.

Much of the petroleum resource potential of Afghanistan and all of the known crude oil and natural gas reserves are in northern Afghanistan, located in parts of two petroliferous geologic basins - the Amu Darya Basin to the west and the Afghan-Tajik Basin to the east (fig. 1). The two basins encompass approximately 515,000 square kilometers (200,000 square miles) in those portions that lie within Afghanistan. Although considerable exploration has previously occurred, both the Amu Darya and Afghan-Tajik Basins of northern Afghanistan are shown in this study to have potential for additional crude oil and natural gas discoveries. The area with the greatest potential for undiscovered natural gas accumulations is in Upper Jurassic carbonate and reef reservoirs beneath an impermeable salt layer in relatively unexplored parts of northern Afghanistan. The area with the greatest potential for undiscovered crude oil accumulations is in Cretaceous to Paleogene carbonate reservoir rocks associated with thrust faulting and folding in the Afghan-Tajik Basin of northeastern Afghanistan. Four total petroleum systems are identified, and these were subdivided into eight assessment units for the purpose of resource assessment (table 1).

\section{Resource Summary}

The U.S. Geological Survey-Afghanistan Ministry of Mines and Industry Joint Oil and Gas Resource Assessment Team assessed undiscovered conventional, technically recoverable petroleum (discovered reserves not included), resulting in the estimated mean volumes of a probability distribution of 1,596 million barrels or 219 million metric tons of crude oil, 15,687 billion cubic feet or 444 billion cubic meters of natural gas, and 562 million barrels or 77 million metric tons of natural gas liquids (table 1). The greatest volume of undiscovered crude oil is estimated to be in the Afghanistan Eastern Suprasalt Thrusts and Folds Assessment Unit (11560202). The greatest volume of undiscovered natural gas is estimated to be in the Afghanistan Jurassic Evaporite Basin Subsalt Carbonates Assessment Unit (11540105). 
Table 1. Northern Afghanistan assessment results (discovered reserves not included).

$[\mathrm{MMB}$, million barrels. BCF, billion cubic feet. Results shown are fully risked estimates. For gas fields, all liquids are included under the natural gas liquids (NGL) category. F95 denotes a 95 percent chance of at least the amount tabulated. Other fractiles are defined similarly. Fractiles are additive under the assumption of perfect positive correlation. TPS, total petroleum system. AU, assessment unit. Gray shading indicates not applicable]

\begin{tabular}{|c|c|c|c|c|c|c|c|c|c|c|c|c|c|}
\hline \multirow{2}{*}{$\begin{array}{l}\text { Total Petroleum Systems } \\
\text { (TPS) } \\
\text { and Assessment Units (AU) } \\
\end{array}$} & \multirow{2}{*}{$\begin{array}{l}\text { Field } \\
\text { Type }\end{array}$} & \multicolumn{12}{|c|}{ Total Undiscovered Resources } \\
\hline & & F95 & F50 & F5 & Mean & F95 & F50 & F5 & Mean & F95 & $\mathrm{F} 50$ & F5 & Mean \\
\hline \multicolumn{14}{|c|}{\begin{tabular}{|l|} 
AMU DARYA BASIN PROVINCE (1154) \\
Amu Darya Jurassic-Cretaceous TPS (115401) \\
\end{tabular}} \\
\hline \multirow{2}{*}{$\begin{array}{l}\text { Afghanistan Jurassic Evaporite } \\
\text { Basin Subsalt Carbonates AU } \\
\text { (11540105) }\end{array}$} & Oil & 0 & 0 & 0 & 0 & 0 & 0 & 0 & 0 & 0 & 0 & 0 & 0 \\
\hline & Gas & & & & & 1,651 & 4,925 & 11,226 & 5,474 & 41 & 128 & 315 & 146 \\
\hline \multirow{2}{*}{$\begin{array}{l}\text { Afghanistan Jurassic Evaporite } \\
\text { Basin Suprasalt Anticlines AU } \\
(11540106)\end{array}$} & Oil & 0 & 0 & 0 & 0 & 0 & 0 & 0 & 0 & 0 & 0 & 0 & 0 \\
\hline & Gas & & & & & 0 & 30 & 1,330 & 324 & 0 & 0 & 22 & 5 \\
\hline \multirow{2}{*}{$\begin{array}{l}\text { Afghanistan Jurassic Evaporite } \\
\text { Basin Margin AU (11540107) }\end{array}$} & Oil & 23 & 67 & 141 & 73 & 21 & 66 & 150 & 73 & 1 & 4 & 9 & 4 \\
\hline & Gas & & & & & 299 & 1,123 & 2,595 & 1,246 & 7 & 29 & 72 & 33 \\
\hline \multicolumn{14}{|c|}{ Kalaimor-Kaisar Jurassic TPS (115402) } \\
\hline \multirow{2}{*}{$\begin{array}{l}\text { Afghanistan Kushka Zone AU } \\
(11540201)\end{array}$} & 0 il & 0 & 0 & 0 & 0 & 0 & 0 & 0 & 0 & 0 & 0 & 0 & 0 \\
\hline & Gas & & & & & 135 & 398 & 869 & 436 & 2 & 6 & 15 & 7 \\
\hline $\begin{array}{l}\text { Total undiscovered petroleum } \\
\text { resources, Amu Darya Basin }\end{array}$ & & 23 & 67 & 141 & 73 & 2,106 & 6,542 & 16,170 & 7,553 & 51 & 167 & 433 & 195 \\
\hline \multicolumn{14}{|c|}{$\begin{array}{l}\text { AFGHAN-TAJIK BASIN PROVINCE (1156) } \\
\text { Afghan-Tajik Jurassic TPS (115601) }\end{array}$} \\
\hline \multirow{2}{*}{$\begin{array}{l}\text { Afghanistan Subsalt Oxfordian } \\
\text { Shelf AU (11560101) }\end{array}$} & Oil & 0 & 0 & 0 & 0 & 0 & 0 & 0 & 0 & 0 & 0 & 0 & 0 \\
\hline & Gas & & & & & 0 & 1,037 & 3,470 & 1,249 & 0 & 15 & 54 & 19 \\
\hline \multirow{2}{*}{$\begin{array}{l}\text { Afghanistan Subsalt Basinal } \\
\text { Facies AU (11560102) }\end{array}$} & Oil & 0 & 0 & 0 & 0 & 0 & 0 & 0 & 0 & 0 & 0 & 0 & 0 \\
\hline & Gas & & & & & 0 & 0 & 3,006 & 778 & 0 & 0 & 47 & 12 \\
\hline \multicolumn{14}{|c|}{ Afghan-Tajik Paleogene TPS (115602) } \\
\hline \multirow{2}{*}{$\begin{array}{l}\text { Afghanistan Western Suprasalt } \\
\text { Gentle Folds AU (11560201) }\end{array}$} & Oil & 89 & 470 & 1,803 & 637 & 308 & 1,630 & 6,370 & 2,234 & 17 & 96 & 385 & 134 \\
\hline & Gas & & & & & 83 & 260 & 760 & 319 & 2 & 5 & 16 & 7 \\
\hline \multirow{2}{*}{$\begin{array}{l}\text { Afghanistan Eastern Suprasalt } \\
\text { Thrusts and Folds AU (11560202) }\end{array}$} & Oil & 279 & 848 & 1,615 & 886 & 954 & 2,938 & 5,781 & 3,098 & 53 & 170 & 370 & 186 \\
\hline & Gas & & & & & 130 & 415 & 905 & 456 & 3 & 8 & 20 & 9 \\
\hline $\begin{array}{l}\text { Total undiscovered petroleum } \\
\text { resources, Afghan-Tajik Basin }\end{array}$ & & 368 & 1,318 & 3,418 & 1,523 & 1,475 & 6,280 & 20,292 & 8,134 & 75 & 294 & 892 & 367 \\
\hline $\begin{array}{l}\text { Total undiscovered petroleum } \\
\text { resources, N. Afghanistan }\end{array}$ & & 391 & 1,385 & 3,559 & 1,596 & 3,581 & 12,822 & 36,462 & 15,687 & 126 & 461 & 1,325 & 562 \\
\hline
\end{tabular}

\section{Further Information}

Supporting geologic studies of total petroleum systems and assessment units, and reports on the methodology used in the North Afghanistan petroleum resource assessment, are in progress. Assessment results are available at the USGS Central Energy Team website: http://energy.cr.usgs.gov/oilgas.

\footnotetext{
${ }^{1}$ U.S. Geological Survey, Box 25046, Denver Federal Center, MS 939, Denver, CO 80225 U.S.A
}

\section{U.S. Geological Survey-Afghanistan Ministry of Mines and Industry Joint Oil and Gas Resource Assessment Team}

T.R. Klett ${ }^{1}$ (Team Leader; tklett@ $@$ usgs.gov), Gregory F. Ulmishek, ${ }^{1}$ C.J. Wandrey ${ }^{1}$ (Project Chief), Warren F. Agena, ${ }^{1}$ David J. Taylor, ${ }^{1}$ Ronald Hill, ${ }^{1}$ Michael Pribil,${ }^{1}$ J. David King, ${ }^{1}$ Mark J. Pawlewicz, ${ }^{1}$ Charles E. Barker, ${ }^{1}$ Thomas S. Ahlbrandt, Ronald R. Charpentier, ${ }^{1}$ Richard M. Pollastro, ${ }^{1}$ Christopher J. Schenk $^{1}$ 